\title{
Determination of antiprotozoal drug mechanisms by metabolomics approaches
}

\author{
DARREN J. CREEK ${ }^{1,2} *$ and MICHAEL P. BARRETT ${ }^{2}$ \\ ${ }^{1}$ Department of Biochemistry and Molecular Biology, Bio21 Molecular Science and Biotechnology Institute, \\ University of Melbourne, 30 Flemington Rd, Parkville, Victoria 3010, Australia \\ ${ }^{2}$ Wellcome Trust Centre for Molecular Parasitology, Institute of Infection, Immunity and Inflammation, and \\ Glasgow Polyomics, College of Medical, Veterinary and Life Sciences, University of Glasgow, Glasgow G12 8TA, UK
}

(Received 17 Fanuary 2013; revised 3 May 2013; accepted 3 May 2013; first published online 5 fune 2013)

\section{SUMMARY}

The discovery, development and optimal utilization of pharmaceuticals can be greatly enhanced by knowledge of their modes of action. However, many drugs currently on the market act by unknown mechanisms. Untargeted metabolomics offers the potential to discover modes of action for drugs that perturb cellular metabolism. Development of high resolution LC-MS methods and improved data analysis software now allows rapid detection of drug-induced changes to cellular metabolism in an untargeted manner. Several studies have demonstrated the ability of untargeted metabolomics to provide unbiased target discovery for antimicrobial drugs, in particular for antiprotozoal agents. Furthermore, the utilization of targeted metabolomics techniques has enabled validation of existing hypotheses regarding antiprotozoal drug mechanisms. Metabolomics approaches are likely to assist with optimization of new drug candidates by identification of drug targets, and by allowing detailed characterization of modes of action and resistance of existing and novel antiprotozoal drugs.

Key words: metabolomics, mode of action, Trypanosoma brucei, protozoan parasite, Plasmodium falciparum, antiprotozoals.

\section{INTRODUCTION}

Protozoan parasites, including Plasmodium spp., Trypanosoma spp. and Leishmania spp., are responsible for diseases that cause significant morbidity, mortality and economic burden, predominantly in developing countries. There are currently no effective vaccines available for the prevention of these tropical diseases, including malaria, human African trypanosomiasis (HAT), Chagas disease and leishmaniasis, and therapy relies heavily on antiprotozoal drugs (Pink et al. 2005; Anthony et al. 2012; Barrett and Croft, 2012). New antiprotozoals are urgently required, as most existing drugs suffer from one or more liabilities related to poor efficacy, drug resistance, toxicity, high cost or unsuitable pharmacokinetic properties (Wells et al. 2009; Phillips, 2012).

The lack of suitable treatments for these tropical diseases is partly due to there having been inadequate pharmaceutical research efforts during the last century (Renslo and McKerrow, 2006; Wells et al. 2009). The majority of current antiprotozoal drugs did not arise from programmes common in contemporary pharmaceutical development, and the rigorous preclinical and clinical evaluation that is now expected

* Corresponding author. Department of Biochemistry and Molecular Biology, Bio21 Molecular Science and Biotechnology Institute, University of Melbourne, 30 Flemington Rd, Parkville, Victoria 3010, Australia. Tel: (+61) 38344 2351. Fax: (+61) 39348 1421. E-mail: darrencreek@gmail.com of new drugs did not accompany registrations of most of the antiprotozoals (Renslo and McKerrow, 2006). Indeed, many commonly used antiprotozoal drugs act by unknown mechanisms, which severely impedes optimal clinical utilization and monitoring for efficacy, toxicity and resistance.

Fortunately, the pipeline for new antiprotozoal drugs now looks promising as a result of recent efforts to screen large compound libraries against these pathogenic parasites. Hundreds of 'hit' compounds have been identified that inhibit the in vitro proliferation of specific protozoan parasites (Gamo et al. 2010; Guiguemde et al. 2010; Duffy and Avery, 2012; Sykes et al. 2012). It is clear that screening compound libraries against organisms is superior to primary screens against individual targets, possibly due to the fact that key pharmacological criteria such as membrane-permeability are already built into compounds passing the former type of screen. A major bottleneck in the hit-to-lead optimization pipeline following identification of hits in whole organism screens relates to the lack of mechanistic information concerning their modes of action (Guiguemde et al. 2012). Identification of target(s) for hit compounds allows rational medicinal chemistry to improve selectivity of binding to the parasite target, and ensures that structural modifications to enhance the pharmacokinetic and toxicity profiles are not likely to compromise activity. The availability of methods to identify modes of action for antiprotozoal compounds in an untargeted manner would greatly 
enhance the efficiency of drug discovery for parasitic diseases.

Metabolomics is an emerging technology that provides an untargeted overview of cellular metabolism by the simultaneous detection and relative quantification of hundreds of small molecules $(<1500 \mathrm{Da})$ in a biological system (Scalbert et al. 2009; Scheltema et al. 2010; Creek et al. 2012a; Dunn et al. 2012). Metabolomic profiling of protozoan parasites treated with antiprotozoal compounds can detect druginduced changes to parasite metabolism and identify the individual metabolites and pathways that are directly perturbed. This provides a rapid and unbiased method to discover likely drug targets (Beyoğlu and Idle, 2013).

The untargeted nature of metabolomics has allowed rapid and unbiased classification of numerous antimicrobial compounds according to their modes of action (Gao et al. 2007; Liu et al. 2009; Halouska et al. 2012). Investigation of the metabolic response of Staphylococcus aureus to triphenylbismuthdichloride revealed pyruvate dehydrogenase as a target for this novel antibiotic (Birkenstock et al. 2012). A more detailed elucidation of the mechanism of antibacterial action was demonstrated by isotopelabelled metabolic flux profiling of Escherichia coli following antifolate exposure (Kwon et al. 2008). Trimethoprim is a known inhibitor of dihydrofolate reductase (DHFR), which was confirmed by accumulation of oxidized folates in the treated cells. However, an unexpected inhibiton of folylpolygamma-glutamate-synthetase was also observed; a response to dihydrofolate accumulation (Kwon et al. 2008). This systems-based approach allows observation of pharmacological effects that often go unnoticed in classical biochemical or genetic approaches based on individual candidate drug targets. Here we discuss the development of platforms that have enabled measurement of hundreds of metabolites in protozoa and their application to drug-target identification.

\section{METABOLOMICS METHODOLOGY}

Metabolomics studies fall into two broad categories, targeted and untargeted. Targeted studies involve hypothesis-driven experiments that aim to provide accurate quantification of a subset of known metabolites, usually restricted to a particular metabolic pathway or chemical class. Untargeted studies by contrast are usually hypothesis-generating experiments that provide relative quantification of all detectable metabolite signals, with subsequent identification of the most significant metabolites (Dunn et al. 2012). Untargeted studies are particularly attractive for the investigation of drugs of unknown mode of action. Targeted studies inherently provide less coverage of the metabolome, but have the advantage of enabling accurate quantification as well as flux

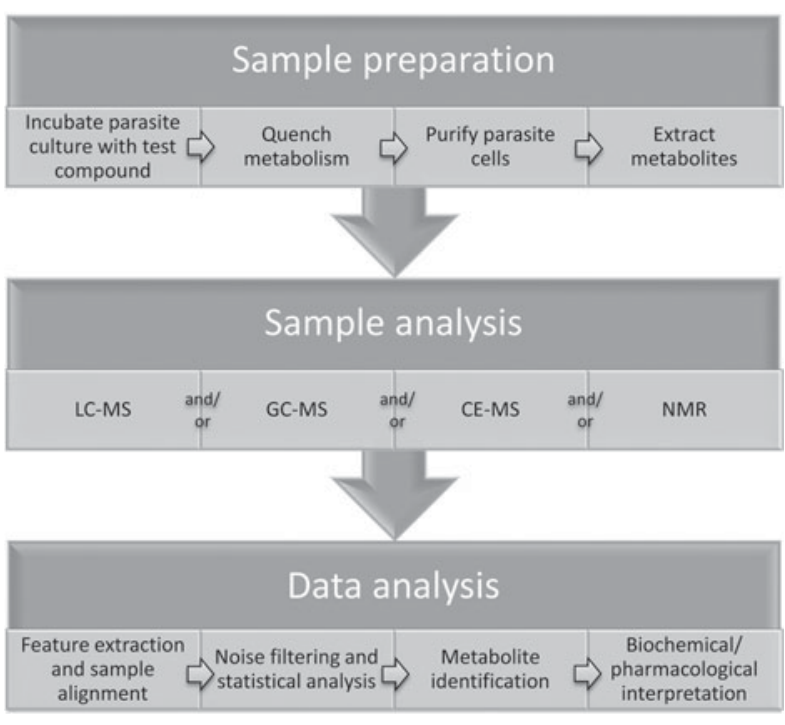

Fig. 1. General outline of methodology for metabolomics studies of protozoan parasites in cell culture.

analysis to determine dynamic responses in candidate pathways (Kwon et al. 2008).

\section{Sample preparation}

Measurements of metabolic responses to drugs are often obtained from in vitro cell culture systems (Fig. 1). This approach allows maximum control over genetic and environmental variables, such as temperature, $\mathrm{pH}$, atmosphere, extracellular environment and cell density, which can contribute to unwanted metabolic alterations and obscure the desired observation of drug-specific effects (Cuperlovic-Culf et al. 2010). The choice of culture media may interfere with drug action and/or metabolic responses, particularly when a standard culture medium does not mimic the in vivo environment of the parasite. For example, the high folate levels present in standard Trypanosoma brucei culture medium, HMI11 (Hirumi and Hirumi, 1989), inhibits the in vitro activity of antifolate compounds that demonstrate trypanocidal activity in vivo (Sienkiewicz et al. 2008), suggesting that culture media should be modified to more closely reflect in vivo conditions wherever possible. In addition, the impact of drug treatment on parasite growth and viability can often alter metabolite abundances, independent of the molecular target of the drug. Multiple controls, time courses and drug concentrations are often necessary to delineate mechanism-related metabolic alterations from non-specific stress responses (Vincent et al. 2012).

Intracellular parasites present unique challenges, and isolation of parasites from uninfected host cells should be performed to avoid interference from host cell metabolites (e.g. saponin lysis or MACS purification for Plasmodium falciparum) (Zhang et al. 2011; Biagini et al. 2012), although it is intriguing to 
consider how impacts on host cell metabolism can contribute to the pharmacological effect of a drug. For example, human cytomegalovirus was shown to lead to up-regulation of host cell fatty acid biosynthesis and inhibitors of fatty acid biosynthesis could inhibit viral growth (Munger et al. 2008). Metabolomic analysis of uninfected host-cell response to drug treatment is also recommended to confirm the parasite-specificity of drug-induced metabolic changes. Care must be taken to avoid metabolic perturbation during any purification step, and metabolic quenching is often achieved by rapid cooling to $0{ }^{\circ} \mathrm{C}$ (Saunders et al. 2011). Following quenching and isolation of cells, metabolites are generally extracted with organic solvent mixtures such as chloroform/ methanol/water (1:3:1) (Scheltema et al. 2010; t'Kindt et al. 2010b).

\section{Sample analysis}

Metabolomics analysis is generally performed with mass spectrometry or NMR spectroscopy. Mass spectrometry is usually coupled to chromatographic separation such as gas chromatography (GC-MS), capillary electrophoresis (CE-MS) or liquid chromatography (LC-MS) (Dunn et al. 2005; Scalbert et al. 2009; Scheltema et al. 2010; Creek et al. 2012a).

LC-MS with high resolution mass spectrometry is becoming the most widely used metabolomics platform due to its high sensitivity, broad specificity and the ability to annotate or identify hundreds of polar and non-polar metabolites from a wide range of metabolic classes ('Theodoridis et al. 2012). Reversed phase chromatography is well established, and is particularly suitable for analysis of lipids and other non-polar metabolites (Theodoridis et al. 2012). Hydrophilic interaction chromatography (HILIC) is finding increasing application in metabolomics, with particular advantages for the separation and identification of polar metabolites (Cubbon et al. 2009; Zhang et al. 2012). High resolution mass spectrometry is critical for the detection and identification of metabolites in untargeted studies. Recent advances in mass spectrometry have enabled widespread application of untargeted metabolomics, as most new time-of-flight (TOF) and Fourier transform (Orbitrap and FT-ICR) mass spectrometers routinely achieve the required resolution $(>30000)$ and mass accuracy ( $<5 \mathrm{ppm}$ ) (Dunn et al. 2012; Theodoridis et al. 2012).

\section{Data analysis}

Computational approaches to metabolomics data analysis are essential, as modern metabolomics platforms routinely detect tens of thousands of ion signals per sample. Many detected peaks arise from noise, contaminants and LC-MS artefacts, and extensive data processing is necessary to allow meaningful interpretation of results (Jankevics et al. 2011; Kuhl et al. 2011; Creek et al. 2012b; Weber et al. 2012). Numerous open-source software packages are available to provide high-throughput detection, alignment, quantification, filtering and/or identification of metabolite signals, including XCMS, Metalign, MZmine, mzMatch and IDEOM (Smith et al. 2006; Lommen, 2009; Pluskal et al. 2010; Scheltema et al. 2011; Creek et al. 2012b). Pre-processed metabolomics data provide relative metabolite abundances for hundreds to thousands of putative metabolites for interrogation by univariate or multivariate statistics (Liland, 2011; Vinaixa et al. 2012).

The major bottleneck for interpretation of untargeted metabolomics data is metabolite identification (Dunn et al. 2012). Many metabolites can be putatively annotated by matching accurate masses to the exact mass of metabolites in biochemical databases. However, accurate metabolite identification is often hampered by metabolites with the same (isomers) or similar (within the error margin of the MS) mass (Scheltema et al. 2010). Confirmation of identification requires orthogonal data such as fragmentation spectra (MS/MS) and chromatographic retention time, and should be compared to authentic standards (Sumner et al. 2007). Unfortunately, many metabolite standards are not easily accessible, and detected metabolites are often reported as putative, or unknown, identities. Isolation and characterization of unidentified metabolites is labour intensive, and is generally only performed for a few metabolites that show statistical significance in a particular study.

Advanced biochemical interpretation of metabolomics studies can be achieved by software solutions that allow mapping of metabolite levels on to metabolic pathways (Paley and Karp, 2006; Jourdan et al. 2010; Leader et al. 2011; Yamada et al. 2011). The bottlenecks associated with metabolite identification limit the usefulness of these approaches, and pathway-based analyses are best suited to targeted metabolomics studies. A compromise approach, which combines untargeted metabolomics with retention time data from authentic standards to improve the confidence of putative metabolite identifications (Creek et al. 2011), is supported by the IDEOM software (Creek et al. 2012b). Interpretation of data in the context of metabolic pathways may also improve the accuracy of metabolite identification (Rogers et al . 2009; Weber and Viant, 2010).

METABOLOMICS FOR INVESTIGATING THE MODE OF ACTION OF ANTIPROTOZOAL DRUGS

\section{Trypanocidal compounds}

$T$. brucei is the causative agent of HAT, otherwise known as sleeping sickness. Infection occurs following the bite of a Tsetse fly, and parasites remain in the bloodstream during stage 1 disease, where symptoms 
are minor and non-specific (Barrett et al. 2007). Stage 2 disease occurs after parasites enter the central nervous system, leading to neurological complications including disturbed sleep-wake patterns. The infection is inevitably fatal if untreated. Five compounds are approved for the treatment of HAT, but each has serious limitations (Brun et al. 2011; Barrett and Croft, 2012). Suramin and pentamidine must be given by injection and are only effective during stage 1 disease because they do not penetrate the bloodbrain barrier. Melarsoprol is active against both stage 1 and stage 2 disease, but this arsenical compound is particularly toxic, with $5-10 \%$ of patients suffering from reactive encephalopathy, which is often fatal. Eflornithine alone, or in combination with nifurtimox is also active against stage $2 \mathrm{CNS}$ disease (Barrett et al. 2007; Phillips, 2012). Clinical utilization of eflornithine may be limited by the requirement for administration of large doses by intravenous infusion, and there is concern that resistance to these drugs may develop rapidly (Vincent et al. 2010). Eflornithine is the only compound among the five approved drugs that has a well-defined mode of action, which involves inhibition of ornithine decarboxylase (ODC) and subsequent depletion of polyamines (Grishin et al. 1999).

The mode of action of eflornithine was recently confirmed using untargeted metabolomics, providing a proof of concept that untargeted metabolomic profiling of protozoan cell cultures is a powerful tool for the unbiased determination of mode of action for antiprotozoal compounds (Vincent et al. 2012). Eflornithine induced significant accumulation of ornithine (the substrate for ODC), and depletion of putrescine (the product of ODC), consistent with direct inhibition of ODC by the drug (Fig. 2). The only other metabolites to consistently show marked changes in abundance were the acetylated forms of ornithine and putrescine. This study also demonstrated a significant depletion of spermidine, the downstream product of the polyamine pathway. Interestingly, only minor down-regulation was observed for trypanothione, the spermidine-bis-glutathione conjugate responsible for antioxidant activity in trypanosomatids, suggesting that the trypanocidal action of eflornithine is more likely related to polyamine depletion than disruption of the antioxidant defence system. Additional metabolite changes were detected after $48 \mathrm{~h}$ incubation with higher drug concentrations, highlighting the importance of metabolomic measurements across a doserange and time-course to delineate direct drug mechanisms from downstream metabolic responses that may include non-specific cell death pathways (Vincent et al. 2012).

Metabolomic analysis of nifurtimox-treated cells revealed a complex pattern of metabolic perturbations, which could not be interpreted as inhibition of a single metabolic enzyme (Vincent et al. 2012).
Altered abundances of nucleotide and glycolytic metabolites were consistent with previous hypotheses that implicate nucleic acid interactions and oxidative stress as likely mediators of nifurtimox activity. One advantage of the untargeted approach was the detection of nifurtimox metabolites that support the likely activation mechanism by $T$. brucei type- 1 nitroreductase (Hall et al. 2011). In contrast to previous hypotheses, nifurtimox was found to lack synergistic activity with eflornithine, and metabolomics data support this lack of synergism (Vincent et al. 2012).

The application of metabolomics to experimental trypanocidal compounds can provide a method to elucidate attractive drug targets for future drug discovery. The impact of fluorinated pyrimidines on T. brucei metabolism was recently described (Ali et al. 2013). 5-fluoro-2'-deoxyuridine resulted in specific accumulation of dUMP in wild-type cells, and minimal change in resistant cells, demonstrating inhibition of thymidylate synthase, most likely by the active metabolite, 5-fluoro-dUMP. In contrast, 5 -fluorouracil and 5 -fluoroorotic acid were incorporated into the uracil nucleotide pool and then into parasite RNA, revealing a distinct mode of action for the fluoropyrimidines compared to the fluorodeoxynucleoside (Ali et al. 2013). The metabolomics platform has recently been applied to a variety of currently used and experimental trypanocidal drugs and work is following up on potential modes of action identified in this way.

\section{Antileishmania compounds}

Leishmania spp. are trypanosomatid parasites responsible for a spectrum of diseases that include visceral, cutaneous and mucocutaneous manifestations of leishmaniasis. The infection is transmitted by sand-fly bite, and parasites develop mostly within macrophages of the mammalian host (Naderer and McConville, 2008). Treatment options for leishmaniasis are severely limited, and the commonly used treatments, pentavalent antimonials, amphotericin B, miltefosine and paromomycin, have poor safety, efficacy and pharmacokinetic profiles (Barrett and Croft, 2012). The mode of action of paromomycin has been investigated by proteomics and, like other aminoglycosides, appears to act by inhibition of protein synthesis (Chawla et al. 2011). Amphotericin $\mathrm{B}$ and miltefosine are thought to interact with sterols and phospholipid metabolism, respectively (Lux et al. 2000; Ouellette et al. 2004; Singh et al. 2012). It is expected that metabolomic or lipidomic studies would be able to confirm the impact of these drugs on lipid metabolism pathways to further characterize the modes of action and resistance.

The mode of action of antimonial compounds is a matter of debate, and is thought to be mediated primarily by oxidative stress. A metabolomics 


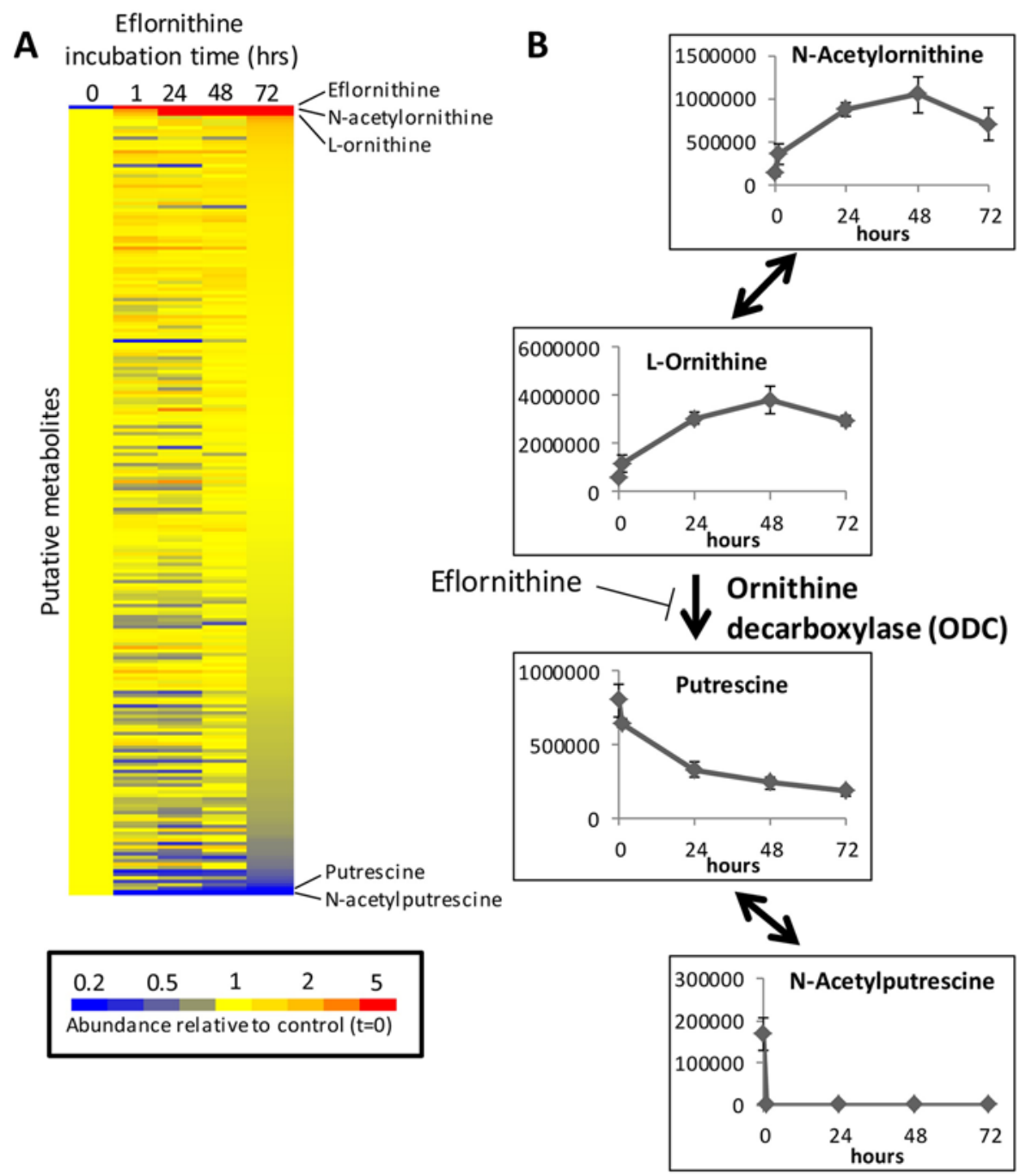

Fig. 2. Metabolomic response of Trypanosoma brucei to eflornithine over a $72 \mathrm{~h}$ incubation time-course (Vincent $e t$ al. 2012). (A) Heat map shows the relative change in abundance of all putative metabolites following incubation with eflornithine. Putative metabolites are ranked according to fold-change at $72 \mathrm{~h}$. The most significant increases and decreases correspond to metabolites of the polyamine pathway. (B) Metabolite abundance profiles of polyamine pathway metabolites showing accumulation of metabolites upstream of ornithine decarboxylase (ODC), and depletion of downstream metabolites. Y-axes represent peak heights from LC-MS data.

approach using CE-MS demonstrated significant perturbation of sulphur-containing amino acids and polyamine pathway metabolites following treatment of Leishmania infantum promastigotes with $\mathrm{Sb}$ (III) (Canuto et al. 2012). This finding is consistent with depletion of trypanothione, the main metabolite responsible for redox homeostasis in trypanosomatids. The mode of resistance to antimonials has also been studied by metabolomics with LC-MS, which revealed significant alterations in the membrane lipid composition of resistant $L$. donovani field isolates (t'Kindt et al. 2010a).

\section{Antimalarial compounds}

Plasmodium spp. are the infectious agents responsible for malaria, with $P$. falciparum and $P$. vivax the most common causative agents of human disease. Plasmodium parasites undergo a complex life-cycle, and are spread by the bite of an infectious mosquito, with the symptomatic phase of disease occurring during replication of asexual parasites within red blood cells of the human host. Numerous drugs are approved for treatment of malaria, although resistance has emerged to most antimalarials (Dondorp et al. 2009; White, 2012). Artemisinin combination therapies (ACTs) are currently recommended due to their potent activity and limited evidence of clinical resistance to date (Anthony et al. 2012; Meshnick, 2012). Nevertheless, new antimalarials are urgently required, as ACTs suffer from emerging resistance, high cost and a pharmacokinetic mismatch between short-acting artemisinins and long-acting partner drugs, usually quinolines (Nosten and White, 2007; Anthony et al. 2012). Artemisinins are thought to act 
by production of free radicals following exposure of the peroxide pharmacophore to $\mathrm{Fe}(\mathrm{II})$ iron and/or haem, which is released as a by-product of haemoglobin digestion within the parasite (O'Neill et al. 2010). The quinoline antimalarials are also thought to act by interaction with intraparasitic haem, and preventing its detoxification (Loria et al . 1999). However, the precise mode of action of most antimalarials remains a matter of debate (Olliaro, 2001; O'Neill et al. 2010). The antifolate antimalarials are known to act by inhibition of DHFR and dihydropteroate synthase (DHPS), but the clinical application of these antifolates is limited by widespread resistance. Nevertheless, characterization of the target enzyme, DHFR, from wild-type and resistant parasites, has lead to the design of novel antimalarial leads that exhibit potent activity against the resistant enzyme (Yuthavong et al. 2012). This demonstrates the potential benefit of understanding the targets of current drugs as a paradigm for new drug discovery.

Atovaquone is another antimalarial with a known mode of action, inhibition of the cytochrome $\mathrm{bc}_{1}$ complex in the mitochondrial electron transport chain. This results in loss of the mitochondrial membrane potential and subsequent disruption of pyrimidine synthesis by indirect inhibition of the essential enzyme dihydroorotate dehydrogenase (DHODH). The mode of action of atovaquone has been confirmed by a targeted metabolomics study of $P$. falciparum, which demonstrated significant accumulation of dihydroorotate, the substrate of DHODH, and its precursor, carbamoyl-L-aspartate (Biagini et al.2012). The mode of action for the novel quinolone, CK-2-68, was also confirmed by observation of the same metabolic response (Biagini et al. 2012).

Several compounds are currently under development for the treatment of malaria, and metabolomics studies may play an important role in defining the mode of action of new antimalarials. Fosmidomycin is an antibiotic that acts on Plasmodium parasites by inhibition of the essential non-mevalonate pathway of isoprenoid biosynthesis. A targeted metabolic profiling study of intermediates in the nonmevalonate isoprenoid synthesis pathway confirmed deoxyxylulose phosphate reductoisomerase (DXR) as the molecular target of the drug, and surprisingly, identified a second target in the pathway, methylerythritol phosphate cytidyltransferase (IspD) (Zhang et al. 2011). The accumulation of all metabolites upstream of IspD suggests that inhibition of this enzyme, either directly by fosmidomycin or by accumulation of 2-C-methylerythrose 4-phosphate, is the primary mode of isoprenoid depletion in P. falciparum (Zhang et al. 2011).

The response of $P$. falciparum in vitro cultures to polyamine pathway inhibitors, eflornithine and MDL73811, was investigated by targeted metabolomics combined with transcriptomics and proteomics. Polyamine depletion was confirmed as the primary mode of action, and unexpected accumulation of glutamate metabolites could be explained by transcriptional upregulation of ornithine aminotransferase in response to ornithine accumulation (van Brummelen et al. 2009).

\section{FUTURE DIRECTIONS}

The suitability of metabolomics technology for determination of the mode of action of antiprotozoal compounds has been clearly demonstrated (Table 1). Further developments in technology will be necessary in order to expand the application of this approach efficiently to all the currently available antiprotozoal drugs, and the hundreds of compounds that display antiprotozoal activity in vitro. Whilst improvements in analytical hardware are expected to continue to expand metabolite coverage and increase sensitivity, the major issue limiting the accessibility of high-throughput metabolomics is data analysis. This situation is rapidly improving as evidenced by many recent advances in freely available data analysis software and web servers. However, it is expected that automated identification of metabolites will remain a significant bottleneck for untargeted metabolomics for the foreseeable future (Dunn et al. 2012).

The ability to relate changes in metabolite abundance to drug action remains largely dependent on $a$ priori knowledge of metabolic pathways within the parasite. Unfortunately, many aspects of parasite metabolism have not been fully elucidated, and often rely on bioinformatic reconstructions of metabolic pathways based on genomic homology with other organisms. Many parasite genes are not annotated, or annotated as hypothetical proteins, and it is likely that some drugs will cause perturbations in metabolites from uncharacterized pathways. In these cases, untargeted metabolomics studies of drug action are likely to provide significant new discoveries in basic parasite biochemistry, as has already been demonstrated for polyamine acetylation (Vincent et al. 2012) and pyrimidine metabolism (Ali et al. 2013) in T. brucei, and the two-step DXR reaction in $P$. falciparum isoprenoid biosynthesis (Zhang et al. 2011).

The major advantage of untargeted metabolomics is the ability to identify a drug-mediated response at the biochemical level with no prior knowledge of the mode of action of a particular antiprotozoal compound. This method can be used to classify compounds according to their mode of action based on a metabolic signature and, in many cases, highlight the specific metabolic enzyme or pathway responsible for antiprotozoal activity. The primary limitation of this approach is that some drugs do not act by disruption of metabolic pathways. In addition, drugs may induce non-specific metabolic changes in response 
Table 1. Examples of applications of metabolomics to determine the mode of action of drugs and other compounds with antimicrobial activity

\begin{tabular}{|c|c|c|c|c|}
\hline Organism & Test compound & $\begin{array}{l}\text { Metabolomics } \\
\text { approach }\end{array}$ & Target identified & Reference \\
\hline T. brucei & Eflornithine & $\begin{array}{l}\text { Untargeted LC-MS } \\
\text { (HILIC-Orbitrap) }\end{array}$ & Ornithine decarboxylase & $\begin{array}{l}\text { (Vincent et al. } \\
\text { 2012) }\end{array}$ \\
\hline T. brucei & Nifurtimox & $\begin{array}{l}\text { Untargeted LC-MS } \\
\text { (HILIC-Orbitrap) }\end{array}$ & $\begin{array}{l}\text { Complex: involves } \\
\text { nucleotides, oxidative stress } \\
\text { and an active metabolite }\end{array}$ & $\begin{array}{l}\text { (Vincent et al. } \\
\text { 2012) }\end{array}$ \\
\hline T. brucei & 5-fluoro-2'-deoxyuridine & $\begin{array}{l}\text { Untargeted LC-MS } \\
\text { (HILIC-Orbitrap) }\end{array}$ & $\begin{array}{l}\text { Thymidylate synthase } \\
\text { inhibited } \\
\text { by active metabolite }\end{array}$ & (Ali et al. 2013) \\
\hline T. brucei & $\begin{array}{l}\text { 5-fluoroorotate and } \\
5 \text {-fluorouracil }\end{array}$ & $\begin{array}{l}\text { Untargeted LC-MS } \\
\text { (HILIC-Orbitrap) }\end{array}$ & $\begin{array}{l}\text { RNA modification } \\
\text { (fluorinated uracil residues) }\end{array}$ & (Ali et al. 2013) \\
\hline L. infantum & Antimony (III) & $\begin{array}{l}\text { Untargeted CE-MS } \\
\text { (CE-TOF) }\end{array}$ & $\begin{array}{l}\text { Oxidative stress (sulphur } \\
\text { amino acids and polyamines) }\end{array}$ & $\begin{array}{l}\text { (Canuto et al. } \\
\text { 2012) }\end{array}$ \\
\hline P. falciparum & Atovaquone and CK-2-68 & $\begin{array}{l}\text { Targeted LC-MS } \\
\text { (HILIC-QQQ) }\end{array}$ & $\begin{array}{l}\text { Dihydroorotate } \\
\text { dehydrogenase (via } \\
\text { mitochondrial electron } \\
\text { transport chain) }\end{array}$ & $\begin{array}{l}\text { (Biagini et al. } \\
\text { 2012) }\end{array}$ \\
\hline P. falciparum & Fosmidomycin & $\begin{array}{l}\text { Targeted LC-MS } \\
\text { (ion paired reversed- } \\
\text { phase-QQQ) }\end{array}$ & $\begin{array}{l}\text { Methylerythritol } \\
\text { phosphate cytidyltransferase } \\
\text { (IspD) and deoxyxylulose } \\
\text { phosphate } \\
\text { reductoisomerase (DXR) }\end{array}$ & (Zhang et al.2011) \\
\hline P. falciparum & $\begin{array}{l}\text { Eflornithine with } \\
\text { MDL73811 }\end{array}$ & $\begin{array}{l}\text { Targeted LC-MS } \\
\text { (HILIC-QQQ) }\end{array}$ & Polyamine synthesis & $\begin{array}{l}\text { (van Brummelen } \\
\text { et al. 2009) }\end{array}$ \\
\hline $\begin{array}{l}\text { Staphylococcus } \\
\text { aureus }\end{array}$ & $\begin{array}{l}\text { Triphenylbismuth } \\
\text { dichloride }\end{array}$ & Targeted ${ }^{1} \mathrm{H}$ NMR & Pyruvate dehydrogenase & $\begin{array}{l}\text { (Birkenstock et al. } \\
\text { 2012) }\end{array}$ \\
\hline S. aureus & $\begin{array}{l}\text { Guanidinomethylbenzoates } \\
\text { and guanidinobenzamides }\end{array}$ & Untargeted GC-MS & $\begin{array}{l}\text { Classified as having similar } \\
\text { mechanism to clindamycin }\end{array}$ & (Liu et al. 2009) \\
\hline Escherichia coli & Trimethoprim & $\begin{array}{l}\text { Targeted LC-MS } \\
\text { (HILIC-QQQ) }\end{array}$ & $\begin{array}{l}\text { Dihydrofolate reducatase } \\
\text { and folylpoly- } \gamma \text {-glutamate } \\
\text { synthetase }\end{array}$ & (Kwon et al. 2008) \\
\hline $\begin{array}{l}\text { E. coli } \\
\text { Pseudomonas } \\
\text { aeruginosa }\end{array}$ & 11 common antibiotics & Targeted CE-UV & $\begin{array}{l}\text { Classification of mechanisms } \\
\text { by correlation analysis }\end{array}$ & (Gao et al. 2007) \\
\hline $\begin{array}{l}\text { Mycobacterium } \\
\text { smegmatis }\end{array}$ & $\begin{array}{l}12 \text { drugs and } 3 \\
\text { chemical leads }\end{array}$ & Untargeted ${ }^{1} \mathrm{H}$ NMR & $\begin{array}{l}\text { Classification of mechanisms } \\
\text { by multivariate analysis }\end{array}$ & $\begin{array}{l}\text { (Halouska et al. } \\
\text { 2012) }\end{array}$ \\
\hline M. smegmatis & D-cycloserine & Untargeted ${ }^{1} \mathrm{H}$ NMR & D-alanine-D-alanine ligase & $\begin{array}{l}\text { (Halouska et al. } \\
\text { 2007) }\end{array}$ \\
\hline
\end{tabular}

to stress, or as secondary responses to the initial drug action. Targeted metabolomics approaches are often required in these circumstances to provide a detailed characterization of the temporal and dose-dependent biochemical effects of drugs. Additional studies, such as transcriptomics, may also be required to delineate primary and secondary metabolic responses to drug treatment (van Brummelen et al. 2009). Generally, measurements of RNA or protein levels are unlikely to reveal the primary targets of drugs that inhibit metabolic enzymes, particularly in trypanosomatid parasites that exhibit polycistronic transcription. Nevertheless, transcriptomic and proteomic approaches are likely to detect the secondary cellular responses to drug treatment, which may be relevant to determination of potential resistance mechanisms. A systems biology approach, that combines metabolomics with other functional genomics tools, is a promising avenue to obtain comprehensive descriptions of the modes of action and resistance for antimicrobial drugs. Metabolomics may also be combined with chemical biology (e.g. protein or ligand microarrays (MacBeath et al. 1999) and chemoinformatic methods (e.g. similarity ensemble approach - Keiser et al. 2010) to improve identification of unknown drug targets in an untargeted manner.

Metabolomics methods offer effective tools for the identification and characterization of mechanisms of drug action, which should optimize drug discovery by allowing detailed pre-clinical pharmacokinetic/pharmocodynamic descriptions of antiprotozoal drugs, and have the potential to translate into clinical studies to monitor both host and parasite responses to drug treatment (Beyoğlu and Idle, 2013).

\section{FINANCIAL SUPPORT}

D. J. C. is supported by an NHMRC postdoctoral training fellowship. M. P. B. is partly supported by the Wellcome Trust through The Wellcome Trust Centre for Molecular 
Parasitology, which is supported by core funding from the Wellcome Trust (Grant 085349).

\section{REFERENCES}

Ali, J. A. M., Creek, D. J., Burgess, K., Allison, H. C., Field, M. C., Mäser, P. and De Koning, H.P. (2013). Pyrimidine salvage in Trypanosoma brucei bloodstream forms and the trypanocidal action of halogenated pyrimidines. Molecular Pharmacology 83, 439-453. doi:10.1124/ mol.112.082321

Anthony, M.P., Burrows, J. N., Duparc, S., Moehrle, J. J. and Wells, T. N. (2012). The global pipeline of new medicines for the control and elimination of malaria. Malaria fournal 11, 316. doi:10.1186/1475 2875-11-316.

Barrett, M. P., Boykin, D. W., Brun, R. and Tidwell, R. R. (2007) Human African trypanosomiasis: pharmacological re-engagement with a neglected disease. British Fournal of Pharmacology 152, 1155-1171. doi:10.1038/sj.bjp.0707354

Barrett, M. P. and Croft, S. L. (2012). Management of trypanosomiasis and leishmaniasis. British Medical Bulletin 104, 174-196. doi:10.1093/bmb/ $1 \mathrm{ds} 031$.

Beyoğlu, D. and Idle, J. R. (2013). Metabolomics and its potential in drug development. Biochemical Pharmacology 85, 12-20. doi:10.1016/j. bcp.2012.08.013

Biagini, G. A., Fisher, N., Shone, A.E., Mubaraki, M. A. Srivastava, A., Hill, A., Antoine, T., Warman, A. J., Davies, J., Pidathala, C., Amewu, R. K., Leung, S. C., Sharma, R., Gibbons, P., Hong, D. W., Pacorel, B., Lawrenson, A. S., Charoensutthivarakul, S. Taylor, L., Berger, O., Mbekeani, A., Stocks, P. A., Nixon, G. L. Chadwick, J., Hemingway, J., Delves, M. J., Sinden, R. E. Zeeman, A.-M., Kocken, C. H. M., Berry, N. G., O’Neill, P. M. and Ward, S. A. (2012). Generation of quinolone antimalarials targeting the Plasmodium falciparum mitochondrial respiratory chain for the treatment and prophylaxis of malaria. Proceedings of the National Academy of Sciences, USA 109, 8298-8303. doi:10.1073/pnas.1205651109.

Birkenstock, T., Liebeke, M., Winstel, V., Krismer, B., Gekeler, C. Niemiec, M. J., Bisswanger, H., Lalk, M. and Peschel, A. (2012). Exometabolome analysis identifies pyruvate dehydrogenase as a target for the antibiotic triphenylbismuthdichloride in multiresistant bacterial pathogens. Fournal of Biological Chemistry 287, 2887-2895. doi:10.1074/jbc. M111.288894.

Brun, R., Don, R., Jacobs, R. T., Wang, M. Z. and Barrett, M. P. (2011) Development of novel drugs for human African trypanosomiasis. Future Microbiology 6, 677-691. doi:10.2217/fmb.11.44

Canuto, G. A. B., Castilho-Martins, E. A., Tavares, M. López-Gonzálvez, Á., Rivas, L. and Barbas, C. (2012). CE-ESI-MS metabolic fingerprinting of Leishmania resistance to antimony treatment. Electrophoresis 33, 1901-1910. doi:10.1002/elps.201200007.

Chawla, B., Jhingran, A., Panigrahi, A., Stuart, K. D. and Madhubala, R. (2011). Paromomycin affects translation and vesiclemediated trafficking as revealed by proteomics of paromomycin-susceptible -resistant Leishmania donovani. PLoS ONE 6, e26660. doi:10.1371/journal. pone.0026660.

Creek, D. J., Anderson, J., McConville, M. J. and Barrett, M.P. (2012a). Metabolomic analysis of trypanosomatid protozoa. Molecula and Biochemical Parasitology 181, 73-84. doi:10.1016/j.molbiopara.2011.10.003.

Creek, D. J., Jankevics, A., Breitling, R., Watson, D. G., Barrett, M. P and Burgess, K.E.V. (2011). Toward global metabolomics analysis with hydrophilic interaction liquid chromatography-mass spectrometry improved metabolite identification by retention time prediction. Analytical Chemistry 83, 8703-8710. doi:10.1021/ac2021823.

Creek, D. J., Jankevics, A., Burgess, K. E. V., Breitling, R. and Barrett, M.P. (2012b). IDEOM: an Excel interface for analysis of LC-MS-based metabolomics data. Bioinformatics 28, 1048-1049. doi:10.1093/bioinformatics/bts069.

Cubbon, S., Antonio, C., Wilson, J. and Thomas-Oates, J. (2009). Metabolomic applications of HILIC-LC-MS. Mass Spectrometry Reviews 29, 671-684. doi:10.1002/mas.20252.

Cuperlovic-Culf, M., Barnett, D. A., Culf, A.S. and Chute, I. (2010). Cell culture metabolomics: applications and future directions. Drug Discovery Today 15, 610-621. doi:10.1016/j.drudis.2010.06.012.

Dondorp, A. M., Nosten, F., Yi, P., Das, D., Phyo, A. P., Tarning, J., Lwin, K. M., Ariey, F., Hanpithakpong, W., Lee, S. J., Ringwald, P., Silamut, K., Imwong, M., Chotivanich, K., Lim, P., Herdman, T. An, S. S., Yeung, S., Singhasivanon, P., Day, N. P. J., Lindegardh, N., Socheat, D. and White, N. J. (2009). Artemisinin resistance in Plasmodium falciparum malaria. New England fournal of Medicine 361, 455-467. doi:10.1056/NEJMoa0808859.

Duffy, S. and Avery, V. M. (2012). Development and optimization of novel 384-well anti-malarial imaging assay validated for high-throughput screening. American Fournal of Tropical Medicine and Hygiene 86, 84-92. doi:10.4269/ajtmh.2012.11-0302.

Dunn, W., Bailey, N. and Johnson, H. (2005). Measuring the metabolome: current analytical technologies. Analyst 130, 606-625. doi:10.1039/ B418288J

Dunn, W. B., Erban, A., Weber, R. J.M., Creek, D. J., Brown, M., Breitling, R., Hankemeier, T., Goodacre, R., Neumann, S., Kopka, J. and Viant, M. R. (2013). Mass appeal: metabolite identification in mas spectrometry-focused untargeted metabolomics. Metabolomics 9, 44-66. doi:10.1007/s11306-012-0434-4.

Gamo, F.-J., Sanz, L. M., Vidal, J., de Cozar, C., Alvarez, E. Lavandera, J.-L., Vanderwall, D. E., Green, D. V.S., Kumar, V. Hasan, S., Brown, J. R., Peishoff, C.E., Cardon, L.R. and Garcia-Bustos, J.F. (2010). Thousands of chemical starting points for antimalarial lead identification. Nature 465, 305-310. doi:10.1038/ nature09107.

Gao, P., Shi, C., Tian, J., Shi, X., Yuan, K., Lu, X. and Xu, G. (2007) Investigation on response of the metabolites in tricarboxylic acid cycle of Escherichia coli and Pseudomonas aeruginosa to antibiotic perturbation by capillary electrophoresis. Fournal of Pharmaceutical and Biomedical Analysis 44, 180-187. doi:10.1016/j.jpba.2007.02.015.

Grishin, N. V., Osterman, A. L., Brooks, H.B., Phillips, M. A. and Goldsmith, E. J. (1999). X-ray structure of ornithine decarboxylase from Trypanosoma bruce $i$ : the native structure and the structure in complex with $\alpha$-difluoromethylornithine. Biochemistry 38, 15174-15184. doi:10.1021/ bi9915115.

Guiguemde, W. A., Shelat, A. A., Bouck, D., Duffy, S., Crowther, G. J., Davis, P.H., Smithson, D.C., Connelly, M., Clark, J., Zhu, F., Jiménez-Díaz, M. B., Martinez, M. S., Wilson, E. B., Tripathi, A. K. Gut, J., Sharlow, E. R., Bathurst, I., Mazouni, F. E., Fowble, J. W. Forquer, I., McGinley, P. L., Castro, S., Angulo-Barturen, I., Ferrer, S., Rosenthal, P. J., DeRisi, J. L., Sullivan, D. J., Lazo, J. S., Roos, D.S., Riscoe, M.K., Phillips, M. A., Rathod, P.K. Van Voorhis, W. C., Avery, V.M. and Guy, R. K. (2010). Chemical genetics of Plasmodium falciparum. Nature 465, 311-315. doi:10.1038/ nature09099.

Guiguemde, W. A., Shelat, A. A., Garcia-Bustos, J. F., Diagana, T. T Gamo, F.-J. and Guy, R. K. (2012). Global phenotypic screening for antimalarials. Chemistry and Biology 19, 116-129. doi:10.1016/j.chembiol.2012.01.004.

Hall, B. S., Bot, C. and Wilkinson, S. R. (2011). Nifurtimox activation by trypanosomal type I nitroreductases generates cytotoxic nitrile metabolites. Fournal of Biological Chemistry 286, 13088-13095. doi:10.1074/jbc. M111.230847.

Halouska, S., Chacon, O., Fenton, R. J., Zinniel, D. K., Barletta, R. G. and Powers, R. (2007). Use of NMR metabolomics to analyze the targets of $\mathrm{d}$-cycloserine in Mycobacteria: role of d-alanine racemase. Fournal of Proteome Research 6, 4608-4614. doi:10.1021/pr0704332.

Halouska, S., Fenton, R. J., Barletta, R. G. and Powers, R. (2012) Predicting the in vivo mechanism of action for drug leads using NMR metabolomics. ACS Chemical Biology 7, 166-171. doi:10.1021/cb200348m. Hirumi, H. and Hirumi, K. (1989). Continuous cultivation of Trypanosoma brucei blood stream forms in a medium containing a low concentration of serum protein without feeder cell layers. Fournal of Parasitology 75, 985-989.

Jankevics, A., Merlo, M. E., de Vries, M., Vonk, R., Takano, E. and Breitling, R. (2011). Separating the wheat from the chaff: a prioritisation pipeline for the analysis of metabolomics datasets. Metabolomics $\mathbf{8}$ (Suppl 1), 29-36. doi:10.1007/s11306-011-0341-0.

Jourdan, F., Cottret, L., Huc, L., Wildridge, D., Scheltema, R. Hillenweck, A., Barrett, M., Zalko, D., Watson, D. and Debrauwer, L. (2010). Use of reconstituted metabolic networks to assist in metabolomic data visualization and mining. Metabolomics 6, 312-321. doi:10.1007/ s11306-009-0196-9.

Keiser, M. J., Irwin, J. J. and Shoichet, B. K. (2010). The chemical basis of pharmacology. Biochemistry 49, 10267.

Kuhl, C., Tautenhahn, R., Böttcher, C., Larson, T. R. and Neumann, S. (2011). CAMERA: an integrated strategy for compound spectra extraction and annotation of liquid chromatography/mass spectrometry data sets. Analytical Chemistry 84, 283-289. doi:10.1021/ac202450g. Kwon, Y.K., Lu, W., Melamud, E., Khanam, N., Bognar, A. and Rabinowitz, J. D. (2008). A domino effect in antifolate drug action in Escherichia coli. Nature Chemical Biology 4, 602-608. doi:10.1038/nchembio. 108 . 
Leader, D. P., Burgess, K., Creek, D. and Barrett, M. P. (2011). Pathos: a web facility that uses metabolic maps to display experimental changes in metabolites identified by mass spectrometry. Rapid Communications in Mass Spectrometry 25, 3422-3426. doi:10.1002/rcm.5245.

Liland, K. H. (2011). Multivariate methods in metabolomics - from preprocessing to dimension reduction and statistical analysis. TrAC Trends in Analytical Chemistry 30, 827-841. doi:10.1016/j.trac.2011.02.007.

Liu, Y., Wen, J., Wang, Y., Li, Y. and Xu, W. (2009). Postulating modes of action of compounds with antimicrobial activities through metabolomics analysis. Chromatographia 71, 253-258. doi:10.1365/s10337-009-1447-7.

Lommen, A. (2009). MetAlign: interface-driven, versatile metabolomics tool for hyphenated full-scan mass spectrometry data preprocessing. Analytical Chemistry 81, 3079-3086. doi:10.1021/ac900036d.

Loria, P., Miller, S., Foley, M. and Tilley, L. (1999). Inhibition of the peroxidative degradation of haem as the basis of action of chloroquine and other quinoline antimalarials. Biochemical fournal 339(Pt 2), 363-370.

Lux, H., Heise, N., Klenner, T., Hart, D. and Opperdoes, F. R. (2000). Ether-lipid (alkyl-phospholipid) metabolism and the mechanism of action of ether-lipid analogues in Leishmania. Molecular and Biochemical Parasitology 111, 1-14. doi:10.1016/S0166-6851(00)00278-4

MacBeath, G., Koehler, A. N. and Schreiber, S. L. (1999). Printing small molecules as microarrays and detecting protein-ligand interactions en masse. Fournal of the American Chemical Society 121, 7967-7968.

Meshnick, S. (2012). Perspective: artemisinin-resistant malaria and the wolf. American Fournal of Tropical Medicine and Hygiene 87, 783-784. doi:10.4269/ajtmh.2012.12-0388

Munger, J., Bennett, B. D., Parikh, A., Feng, X.-J., McArdle, J., Rabitz, H. A., Shenk, T. and Rabinowitz, J. D. (2008). Systems-level metabolic flux profiling identifies fatty acid synthesis as a target for antiviral therapy. Nature Biotechnology 26, 1179-1186. doi:10.1038/nbt.1500.

Naderer, T. and McConville, M. J. (2008). The Leishmania-macrophage interaction: a metabolic perspective. Cellular Microbiology 10, 301-308. doi:10.1111/j.1462-5822.2007.01096.x

Nosten, F. and White, N. J. (2007). Artemisinin-based combination treatment of falciparum malaria. American Fournal of Tropical Medicine and Hygiene 77(6 Suppl), 181-192.

Olliaro, P. (2001). Mode of action and mechanisms of resistance for antimalarial drugs. Pharmacology and Therapeutics 89, 207-219. doi:10.1016/ S0163-7258(00)00115-7.

O'Neill, P. M., Barton, V.E. and Ward, S. A. (2010). The molecular mechanism of action of artemisinin - the debate continues. Molecules $\mathbf{1 5}$, 1705-1721. doi:10.3390/molecules15031705.

Ouellette, M., Drummelsmith, J. and Papadopoulou, B. (2004). Leishmaniasis: drugs in the clinic, resistance and new developments. Drug Resistance Updates 7, 257-266. doi:10.1016/j.drup.2004.07.002.

Paley, S. M. and Karp, P. D. (2006). The Pathway Tools cellular overview diagram and Omics Viewer. Nucleic Acids Research 34, 3771-3778. doi:10.1093/nar/gk1334

Phillips, M.A. (2012). Stoking the drug target pipeline for human African trypanosomiasis. Molecular Microbiology 86, 10-14. doi:10.1111/ mmi.12001.

Pink, R., Hudson, A., Mouries, M.-A. and Bendig, M. (2005). Opportunities and challenges in antiparasitic drug discovery. Nature Reviews Drug Discovery 4, 727-740. doi:10.1038/nrd1824.

Pluskal, T., Castillo, S., Villar-Briones, A. and Oresic, M. (2010). MZmine 2: modular framework for processing, visualizing, and analyzing mass spectrometry-based molecular profile data. BMC Bioinformatics $\mathbf{1 1}$ 395. doi: 10.1186/1471-2105-11-395

Renslo, A. R. and McKerrow, J.H. (2006). Drug discovery and development for neglected parasitic diseases. Nature Chemical Biology 2, 701-710. doi:10.1038/nchembio837.

Rogers, S., Scheltema, R. A., Girolami, M. and Breitling, R. (2009). Probabilistic assignment of formulas to mass peaks in metabolomics experiments. Bioinformatics 25, 512-518. doi:10.1093/bioinformatics/ btn642.

Saunders, E. C., Ng, W. W., Chambers, J. M., Ng, M., Naderer, T., Kramer, J. O., Likic, V. A. and McConville, M. J. (2011). Isotopomer profiling of Leishmania mexicana promastigotes reveals important roles for succinate fermentation and aspartate uptake in tricarboxylic scid cycle (TCA) anaplerosis, glutamate synthesis, and growth. Fournal of Biological Chemistry 286, 27706-27717. doi:10.1074/jbc.M110.213553.

Scalbert, A., Brennan, L., Fiehn, O., Hankemeier, T., Kristal, B., van Ommen, B., Pujos-Guillot, E., Verheij, E., Wishart, D. and Wopereis, S. (2009). Mass-spectrometry-based metabolomics: limitations and recommendations for future progress with particular focus on nutrition research. Metabolomics 5, 435-458. doi:10.1007/s11306-009-0168-0.

Scheltema, R. A., Decuypere, S., t'Kindt, R., Dujardin, J.-C., Coombs, G. H. and Breitling, R. (2010). The potential of metabolomics for Leishmania research in the post-genomics era. Parasitology 137, 1291 1302. doi:10.1017/S0031182009992022.

Scheltema, R. A., Jankevics, A., Jansen, R. C., Swertz, M. A. and Breitling, R. (2011). PeakML/mzMatch: a file format, java library, R library, and tool-chain for mass spectrometry data analysis. Analytical Chemistry 83, 2786-2793. doi:10.1021/ac2000994.

Sienkiewicz, N., Jaroslawski, S., Wyllie, S. and Fairlamb, A. H. (2008). Chemical and genetic validation of dihydrofolate reductasethymidylate synthase as a drug target in African trypanosomes. Molecular Microbiology 69, 520-533. doi:10.1111/1365-2958.2008.06305x.

Singh, N., Kumar, M. and Singh, R. K. (2012). Leishmaniasis: current status of available drugs and new potential drug targets. Asian Pacific fournal of Tropical Medicine 5, 485-497. doi:10.1016/S1995-7645(12)60084-4.

Smith, C., Want, E., O'Maille, G., Abagyan, R. and Siuzdak, G. (2006) XCMS: processing mass spectrometry data for metabolite profiling using nonlinear peak alignment, matching, and identification. Analytical Chemistry 78, 779-787. doi:10.1021/ac051437y

Sumner, L., Amberg, A., Barrett, D., Beale, M., Beger, R., Daykin, C., Fan, T., Fiehn, O., Goodacre, R., Griffin, J., Hankemeier, T., Hardy, N., Harnly, J., Higashi, R., Kopka, J., Lane, A., Lindon, J., Marriott, P., Nicholls, A., Reily, M., Thaden, J. and Viant, M. (2007). Proposed minimum reporting standards for chemical analysis. Metabolomics 3, 211-221. doi:10.1007/s11306-007-0082-2.

Sykes, M. L., Baell, J. B., Kaiser, M., Chatelain, E., Moawad, S. R., Ganame, D., Ioset, J.-R. and Avery, V. M. (2012). Identification of compounds with anti-proliferative activity against Trypanosoma brucei brucei strain 427 by a whole cell viability based HTS campaign. PLoS Neglected Tropical Diseases 6, e1896. doi:10.1371/journal.pntd.0001896. Theodoridis, G.A., Gika, H. G., Want, E.J. and Wilson, I. D. (2012). Liquid chromatography-mass spectrometry based global metabolite profiling: a review. Analytica Chimica Acta 711, 7-16. doi:10.1016/j. aca.2011.09.042.

t'Kindt, R., Jankevics, A., Scheltema, R., Zheng, L., Watson, D., Dujardin, J.-C., Breitling, R., Coombs, G. and Decuypere, S. (2010b). Towards an unbiased metabolic profiling of protozoan parasites: optimisation of a Leishmania sampling protocol for HILIC-orbitrap analysis. Analytical and Bioanalytical Chemistry 398, 2059-2069. doi:10.1007/ s00216-010-4139-0.

t'Kindt, R., Scheltema, R. A., Jankevics, A., Brunker, K., Rijal, S., Dujardin, J.-C., Breitling, R., Watson, D. G., Coombs, G.H. and Decuypere, S. (2010a). Metabolomics to unveil and understand phenotypic diversity between pathogen populations. PLoS Neglected Tropical Diseases 4, e904. doi:10.1371/journal.pntd.0000904.

van Brummelen, A. C., Olszewski, K. L., Wilinski, D., Llinas, M., Louw, A. I. and Birkholtz, L. M. (2009). Co-inhibition of Plasmodium falciparum S-adenosylmethionine decarboxylase/ornithine decarboxylase reveals perturbation-specific compensatory mechanisms by transcriptome, proteome, and metabolome analyses. Fournal of Biological Chemistry 284, 4635-4646. doi:10.1074/jbc. M807085200.

Vinaixa, M., Samino, S., Saez, I., Duran, J., Guinovart, J. J. and Yanes, O. (2012). A guideline to univariate statistical analysis for LC/MSbased untargeted metabolomics-derived data. Metabolites 2, 775-795. doi:10.3390/metabo2040775.

Vincent, I. M., Creek, D., Watson, D. G., Kamleh, M. A., Woods, D. J., Wong, P. E., Burchmore, R. J. S. and Barrett, M. P. (2010). A molecular mechanism for eflornithine resistance in African trypanosomes. PLoS Pathogens 6, e1001204. doi:10.1371/journal.ppat.1001204.

Vincent, I. M., Creek, D. J., Burgess, K., Woods, D. J., Burchmore, R. J. S. and Barrett, M. P. (2012). Untargeted metabolomics reveals a lack of synergy between nifurtimox and eflornithine against Trypanosoma brucei. PLoS Neglected Tropical Diseases 6, e1618. doi:10.1371/journal.pntd.0001618.

Weber, R. J. M. and Viant, M. R. (2010). MI-Pack: increased confidence of metabolite identification in mass spectra by integrating accurate masses and metabolic pathways. Chemometrics and Intelligent Laboratory Systems 104, 75-82. doi:10.1016/j.chemolab.2010.04.010.

Weber, R. J. M., Li, E., Bruty, J., He, S. and Viant, M. R. (2012) MaConDa: a publicly accessible mass spectrometry contaminants database. Bioinformatics 28, 2856-2857. doi:10.1093/bioinformatics/bts527. Wells, T. N. C., Alonso, P.L. and Gutteridge, W.E. (2009). New medicines to improve control and contribute to the eradication of malaria. Nature Reviews Drug Discovery 8, 879-891. doi:10.1038/nrd2972.

White, N. J. (2012). Counter perspective: artemisinin resistance: facts, fears, and fables. American Fournal of Tropical Medicine and Hygiene 87, 785. doi:10.4269/ajtmh.2012.12-0573.

Yamada, T., Letunic, I., Okuda, S., Kanehisa, M. and Bork, P. (2011) iPath2.0: interactive pathway explorer. Nucleic Acids Research 39(Suppl 2), W412-W415. doi:10.1093/nar/gkr313. 
Yuthavong, Y., Tarnchompoo, B., Vilaivan, T., Chitnumsub, P., Kamchonwongpaisan, S., Charman, S.A., McLennan, D. N., White, K.L., Vivas, L., Bongard, E., Thongphanchang, C. Taweechai, S., Vanichtanankul, J., Rattanajak, R., Arwon, U., Fantauzzi, P., Yuvaniyama, J., Charman, W. N. and Matthews, D. (2012). Malarial dihydrofolate reductase as a paradigm for drug development against a resistance-compromised target. Proceedings of the National Academy of Sciences, USA 109, 16823-16828. doi:10.1073/ pnas. 1204556109 .
Zhang, B., Watts, K. M., Hodge, D., Kemp, L. M., Hunstad, D. A., Hicks, L. M. and Odom, A. R. (2011). A second target of the antimalarial and antibacterial agent fosmidomycin revealed by cellular metabolic profiling. Biochemistry 50, 3570-3577. doi:10.1021/bi200113y.

Zhang, T., Creek, D. J., Barrett, M.P., Blackburn, G. and Watson, D. G. (2012). Evaluation of coupling reversed phase, aqueous normal phase, and hydrophilic interaction liquid chromatography with Orbitrap mass spectrometry for metabolomic studies of human urine. Analytical Chemistry 84, 1994-2001. doi:10.1021/ac2030738. 